\title{
Awake perimetry testing for occipital epilepsy surgery
}

\author{
Holger Joswig, MD, FMH, John P. Girvin, MD, PhD, FRCSC, ${ }^{1}$ Warren T. Blume, MD, FRCPC, ${ }^{2}$ \\ Jorge G. Burneo, MD, MSPH, ${ }^{2}$ and David A. Steven, MD, MPH, FRCSC ${ }^{1}$
}

\author{
Epilepsy Program, Divisions of ${ }^{1}$ Neurosurgery and ${ }^{2}$ Neurology, Department of Clinical Neurological Sciences, London Health \\ Sciences Centre, University Hospital, London, Ontario, Canada
}

\begin{abstract}
In the literature, there are few reports that provide a detailed account on the technique of visual electrocortical stimulation in the setting of resective surgery for occipital epilepsy. In this technical note, the authors describe how a 26 -yearold male with long-standing occipital epilepsy underwent resective surgery under awake conditions, using electrocortical stimulation of the occipital lobe, with the aid of a laser pointer and a perimetry chart on a stand within his visual field. The eloquent primary visual cortex was found to overlap with the seizure onset zone that was previously determined with subdural electrodes. A maximum functionally safe resection was performed, rendering the patient seizure free as of his last follow-up at 20 months, with no visual field impairment.
\end{abstract}

https://thejns.org/doi/abs/10.3171/2017.6.JNS17846

KEY WORDS awake surgery; electrocortical stimulation; epilepsy surgery; mapping; occipital epilepsy; perimetry; visual cortex; surgical technique

$\mathrm{O}$ CCIPITAL epilepsy (OE) represents a small fraction of all seizure disorders and can be drug resistant. ${ }^{3}$ However, OE is amenable to resective surgery, with reported long-term seizure freedom in $46 \%-65 \%$ of the cases. ${ }^{6,17}$ The visual status of the patient and whether or to what degree a postoperative visual field deficit is acceptable is a pivotal discussion between the patient and surgeon prior to surgery. ${ }^{5}$ To minimize the likelihood for or extent of postoperative visual impairment, mapping techniques by means of electrocortical stimulation under awake conditions have been applied since the beginning of epilepsy surgery. ${ }^{14}$ Nevertheless, although numerous case series with reports on outcome following resective surgery involving the occipital lobe exist,,$^{1-3,7,8,10,14,18,21}$ only a few mention intraoperative mapping as an adjunct to preserve visual integrity. ${ }^{3,14,21}$ In fact, the literature lacks detailed descriptions of the technique on how to perform visual electrocortical stimulation. In his book Operative Techniques in Epilepsy, ${ }^{5}$ Girvin briefly mentions the use of a visual board, which has been a well-established practice for awake perimetry testing at our institution and will be illustrated in the following case.

\section{Illustrative Case}

A right-hand-dominant 26-year-old man was evaluated for a 10-year history of epilepsy. The patient described daily events that consisted of visual distortion in the form of a fading, blurry vision in his right visual field, occasionally followed by loss of awareness and manual automatisms. Rarely, secondary generalization with postictal word-finding difficulties occurred. The patient's neurological examination, including formal visual field testing, revealed normal findings. Scalp electroencephalography (EEG) revealed abundant epileptiform discharges over T5-occiput with seizure onset from the same area, which rapidly spread to the ipsilateral temporal lobe. MRI findings were normal. To identify the seizure onset zone (SOZ), an invasive EEG study covering the left temporal lobe and regions of the parietal and occipital lobes with subdural electrodes inserted via a suprasinusoidal temporooccipital approach ${ }^{16}$ was carried out (Fig. 1). A precise focus was identified in the left occipital lobe (Fig. 2A, Electrodes 111-114, with early spread to Electrodes 115 and 116). Based on the semiology and invasive EEG recordings, it was hypothesized that the seizures were arising from the left visual association area, rather than the primary visual cortex. In addition, extraoperative stimulation with the subdural electrodes suggested that the SOZ was outside the primary visual cortex. Nonetheless, given the proximity of the SOZ to the primary visual cortex and the fact that the patient

ABBREVIATIONS EEG = electroencephalography; OE = occipital epilepsy; $S O Z$ = seizure onset zone.

SUBMITTED April 3, 2017. ACCEPTED June 13, 2017.

INCLUDE WHEN CITING Published online December 8, 2017; DOI: 10.3171/2017.6.JNS17846. 




FIG. 1. Three-dimensional reconstruction of the subdural electrode coverage of the left temporal lobe $(031-038,051-058)$ and regions of the parietal and occipital lobes $(071-078$ and 091-098, 111-118) via a standard suprasinusoidal temporooccipital approach. Figure is available in color online only.

had normal visual fields on automated visual field testing, we decided to perform the resective surgery under awake conditions with electrocortical stimulation for maximum safe resection of the epileptogenic focus.

\section{Procedure}

The resective procedure was performed 6 months after the subdural electrode study. To plan the resection, a CT scan obtained in the patient with the subdural electrodes in place was merged with an MR image from the day of surgery using the StealthStation neuronavigation software (Medtronic). With the patient in the right lateral decubitus position, a scalp block was performed using a mixture of lidocaine $1 \%$ and bupivacaine $0.5 \%$ with epinephrine $(1: 200,000)$, and the head was fixed in a 3-point headrest. We adhered to our sedation-awake-sedation protocol using propofol/remifentanil. A horseshoe incision was performed and a craniotomy was fashioned to provide generous exposure to the occipital lobe including the areas covered previously by the subdural electrodes.

After exposing the occipital lobe, we performed monopolar electrocortical stimulation with the patient fully awake and cooperative. A large perimetry chart on a stand turned to $90^{\circ}$ was placed in front of the patient for intraoperative mapping of the visual field. The patient was provided with a laser pointer, and, in response to ongoing electrocortical stimulation of several visual cortical areas, he was able to point exactly toward the perimetry chart to acknowledge any positive or negative changes in his vision (Fig. 3). These visual areas were marked on the board, and the corresponding cortex was labeled with letter tags (Fig. 2A). Stimulation in areas presumed to represent primary visual cortex elicited a simple phenomenon of a small distorted "black and white" light, whereas stimulation in areas presumed to represent visual association cortex led to visual distortion. As the eloquent primary visual cortex overlapped with the active Contacts 11 and 12, only cortex corresponding to the active Contacts 13 to 16 was resected (Fig. 2B). The corticectomy was carried out in a subpial fashion, sparing the cortical vessels as well as the underlying white matter. Following resection, closure was done in the usual fashion. The patient tolerated the procedure well, and there were no new neurological deficits after surgery. In particular, there were no visual field deficits on Goldman perimetry testing at 3 months postoperatively. Unfortunately, no specimen was obtained for histopathological assessment.

\section{Follow-Up}

Three months after surgery, the patient experienced one primary generalized tonic-clonic seizure that was not preceded by his typical visual or any other aura and lasted 1 minute, while remaining on a regimen of antiepileptic therapy consisting of lamotrigine $100 \mathrm{mg}$ twice daily and 

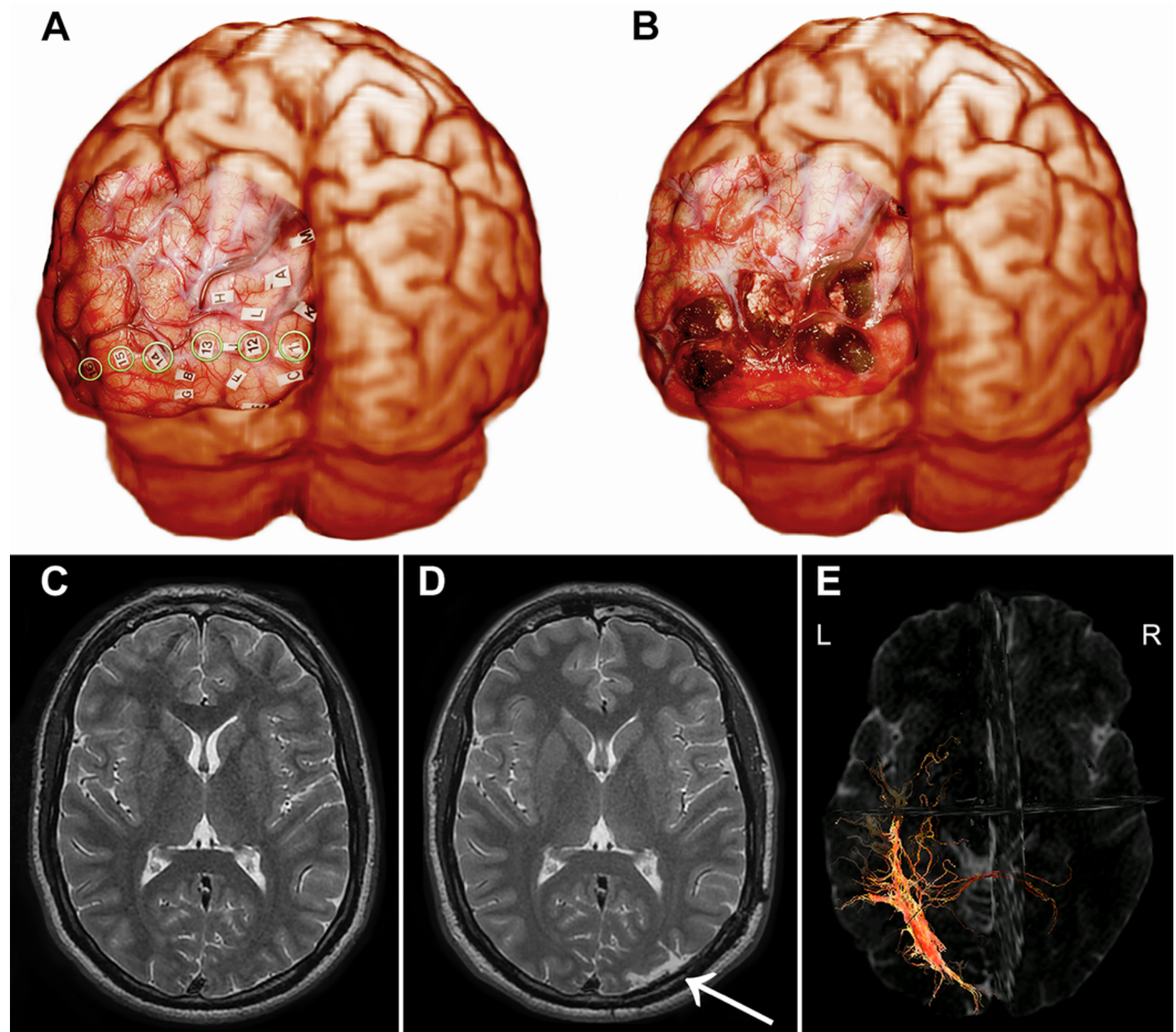

FIG. 2. A: After exposure of the left occipital cortex, monopolar electrocortical stimulation with $3.5 \mathrm{~mA}$ was used to delineate eloquent visual cortex. The letters indicate sites that produced the following: A, "dizzy" sensation with visual distortion and diplopia; B, visual distortion; $\mathrm{C}-\mathrm{G}$ and $\mathrm{K}$, small, distorted, moving "black and white" light; $\mathrm{H}-\mathrm{J}$, broad area of brightness; $\mathrm{L}$ and $\mathrm{M}$, visual distortion and illusion that lines on the perimetry board were moving. Circled numbers represent the location of the subdural electrode contacts. The seizure onset zone included Electrodes 111-114 with early spread to Electrodes 115 and 116. It was interpreted from the above stimulation results that the primary cortex involved Sites $C-G$ and K. Sites A, B, H, J, L, and M were determined to be situated in the secondary visual cortex. B: Postresection photograph. C: Preoperative axial MR image. D: Postoperative axial MR image showing the extent of the left occipital cortical resection (arrow). E: Preoperative diffusion tensor imaging tractography study of the left optic radiation (top view). Figure is available in color online only.

lacosamide $150 \mathrm{mg}$ twice daily. Sixteen months after surgery, the patient discontinued his antiepileptic drugs, yet remained completely seizure free as of his last follow-up visit 20 months postoperatively.

\section{Discussion}

Postoperative visual field integrity plays a major role in elective resective surgery for epilepsy. While the major- ity of cases with Meyer's loop impairment after anterior temporal lobectomy is probably clinically insignificant and further improvement can be observed in one-third of those with large visual field deficits, ${ }^{20}$ preservation or sacrifice of visual function always determines the presurgical considerations and limits the resection extent in OE. ${ }^{3}$ At our institution, the rate of postsurgical visual field deficits was $32 \%$ under intraoperative continuous visual field assessment and electrocorticography-guided resection. ${ }^{3} \mathrm{We}$ 


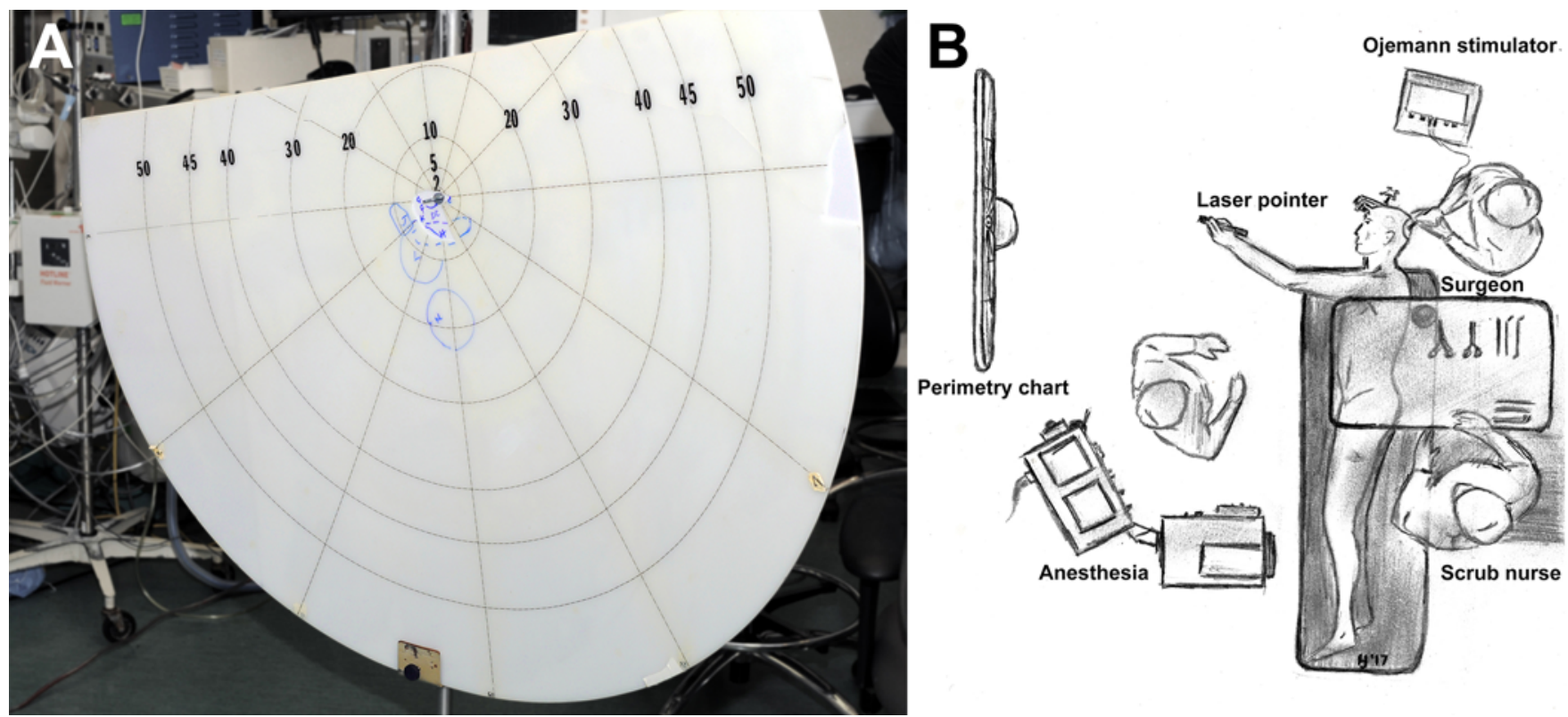

FIG. 3. A: Perimetry chart used for awake perimetry testing during occipital electrocortical stimulation for resection of a left occipital epileptogenic focus. Note that the blue-zoned visual field and letters correspond to the stimulated occipital cortex (compare with the legend for Fig. 2A). B: Intraoperative setup. Copyright London Health Sciences Centre (panel B). Published with permission. Figure is available in color online only.

agree with the notion of Binder et al. ${ }^{2}$ that the outcome of epilepsy surgery in the occipital lobe has significantly increased with the introduction of modern MRI, video-EEG, and invasive monitoring over the years. The use of invasive monitoring by means of intracranial subdural ${ }^{1-3,7,8,10,11,14,21}$ and depth ${ }^{1,2,8,9,14,18,21}$ electrodes to delineate the epileptogenic zone is widespread, as is conducting extraoperative stimulation. ${ }^{1,2,9-11,21}$ In the same vein, functional MRI ${ }^{13}$ and diffusion tensor imaging tractography ${ }^{19}$ have been added to the preoperative diagnostic armamentarium to visualize eloquent cortex with existing visual field defects and optic white matter tracts, respectively (Fig. 2E). Yet warranting further clinical evaluation, these novel technologies have their merits. However, up until now, we regard intraoperative awake perimetry testing as the gold standard to avoid visual field impairment. Intraoperative visual evoked potentials monitoring ${ }^{12}$ or extraoperative mapping could represent valuable alternatives for those patients who cannot undergo or who reject awake surgery.

It should be noted that, in this illustrative case, electrocortical stimulation elicited only simple visual phenomena. In the context of the literature, this is an expected finding. Complexity of elicited visual phenomena increases in a posteroanterior axis and can be categorized into simple (e.g., flashing lights), intermediate (simple geometrical shapes), and complex forms..$^{9,11}$ In particular, the left occipital cortex is known to have a substantially lower sensitivity for stimulation and lacks complex shapes compared with the visually dominant right occipital lobe during extraoperative stimulation using depth electrodes. ${ }^{9}$ Moreover, $67 \%$ of the striate cortex is buried in the calcarine fissure with its branches and accessory sulci ${ }^{15}$ and thus is inaccessible for stimulation.

In essence, this case illustrates how awake perimetry during electrocortical stimulation of the occipital lobe can simply be facilitated with the use of a laser pointer and a perimetry chart on a stand within the visual field of the patient in the operating room. Another way is to just let the patient draw their visual responses out on a white paper. ${ }^{11}$ Practical methods like these are effective and more likely to be successfully implemented in epilepsy surgery centers with less experience with awake stimulations or those with limited resources. ${ }^{4}$

\section{Acknowledgments}

Mr. Frank Bihari co-registered the patient's noncontrast CT scan with an electrode protocol (nonhelical, reformatted to an axial slice thickness of $1.25 \mathrm{~mm}$ ) (for creation of Fig. 1) and by draping and rendering of JPEG intraoperative photograph bitmaps (for creation of Fig. 2A and B) over the surface of the patient's 3D T1-weighted high-resolution SPRG MRI of the brain, respectively, using Atamai Epilepsy Viewer (version 4 14b, 2007).

We thank Dr. Donald Lee and Mr. Douglas Cesarin for creation of Fig. 2E from diffusion tensor tractography MRI.

\section{References}

1. Aykut-Bingol C, Bronen RA, Kim JH, Spencer DD, Spencer SS: Surgical outcome in occipital lobe epilepsy: implications for pathophysiology. Ann Neurol 44:60-69, 1998

2. Binder DK, Von Lehe M, Kral T, Bien CG, Urbach H, Schramm J, et al: Surgical treatment of occipital lobe epilepsy. J Neurosurg 109:57-69, 2008

3. Blume WT, Whiting SE, Girvin JP: Epilepsy surgery in the posterior cortex. Ann Neurol 29:638-645, 1991

4. Burneo JG, Delgado JC, Steven DA, Vasquez CM, AlonsoVanegas MA, Cavazos JE, et al: A collaborative effort to establish a comprehensive epilepsy program in Peru. Epilepsy Behav 26:96-99, 2013 
5. Girvin JP: Operative Techniques in Epilepsy. Cham, Switzerland: Springer, 2015

6. Harward SC, Chen WC, Rolston JD, Haglund MM, Englot DJ: Seizure outcomes in occipital lobe and posterior quadrant epilepsy surgery: a systematic review and meta-analysis. Neurosurgery [epub ahead of print], 2017

7. Ibrahim GM, Fallah A, Albert GW, Withers T, Otsubo H, Ochi A, et al: Occipital lobe epilepsy in children: characterization, evaluation and surgical outcomes. Epilepsy Res 99:335-345, 2012

8. Jobst BC, Williamson PD, Thadani VM, Gilbert KL, Holmes GL, Morse RP, et al: Intractable occipital lobe epilepsy: clinical characteristics and surgical treatment. Epilepsia 51:2334-2337, 2010

9. Jonas J, Frismand S, Vignal JP, Colnat-Coulbois S, Koessler L, Vespignani H, et al: Right hemispheric dominance of visual phenomena evoked by intracerebral stimulation of the human visual cortex. Hum Brain Mapp 35:3360-3371, 2014

10. Kuzniecky R, Gilliam F, Morawetz R, Faught E, Palmer C, Black L: Occipital lobe developmental malformations and epilepsy: clinical spectrum, treatment, and outcome. Epilepsia 38:175-181, 1997

11. Lee HW, Hong SB, Seo DW, Tae WS, Hong SC: Mapping of functional organization in human visual cortex: electrical cortical stimulation. Neurology 54:849-854, 2000

12. Ota T, Kawai K, Kamada K, Kin T, Saito N: Intraoperative monitoring of cortically recorded visual response for posterior visual pathway. J Neurosurg 112:285-294, 2010

13. Roux FE, Ibarrola D, Lotterie JA, Chollet F, Berry I: Perimetric visual field and functional MRI correlation: implications for image-guided surgery in occipital brain tumours. J Neurol Neurosurg Psychiatry 71:505-514, 2001

14. Salanova V, Andermann F, Olivier A, Rasmussen T, Quesney LF: Occipital lobe epilepsy: electroclinical manifestations, electrocorticography, cortical stimulation and outcome in 42 patients treated between 1930 and 1991. Surgery of occipital lobe epilepsy. Brain 115:1655-1680, 1992

15. Stensaas SS, Eddington DK, Dobelle WH: The topography and variability of the primary visual cortex in man. J Neurosurg 40:747-755, 1974

16. Steven DA, Andrade-Souza YM, Burneo JG, McLachlan RS, Parrent AG: Insertion of subdural strip electrodes for the investigation of temporal lobe epilepsy. Technical note. $\mathbf{J}$ Neurosurg 106:1102-1106, 2007
17. Téllez-Zenteno JF, Dhar R, Wiebe S: Long-term seizure outcomes following epilepsy surgery: a systematic review and meta-analysis. Brain 128:1188-1198, 2005

18. Williamson PD, Thadani VM, Darcey TM, Spencer DD, Spencer SS, Mattson RH: Occipital lobe epilepsy: clinical characteristics, seizure spread patterns, and results of surgery. Ann Neurol 31:3-13, 1992

19. Winston GP, Yogarajah M, Symms MR, McEvoy AW, Micallef C, Duncan JS: Diffusion tensor imaging tractography to visualize the relationship of the optic radiation to epileptogenic lesions prior to neurosurgery. Epilepsia 52:1430-1438, 2011

20. Yam D, Nicolle D, Steven DA, Lee D, Hess T, Burneo JG: Visual field deficits following anterior temporal lobectomy: long-term follow-up and prognostic implications. Epilepsia 51:1018-1023, 2010

21. Yang PF, Jia YZ, Lin Q, Mei Z, Chen ZQ, Zheng ZY, et al: Intractable occipital lobe epilepsy: clinical characteristics, surgical treatment, and a systematic review of the literature. Acta Neurochir (Wien) 157:63-75, 2015

\section{Disclosures}

The authors report no conflict of interest concerning the materials or methods used in this study or the findings specified in this paper.

\section{Author Contributions}

Conception and design: Steven, Joswig. Acquisition of data: Steven, Joswig. Analysis and interpretation of data: Steven, Joswig. Drafting the article: Steven, Joswig. Critically revising the article: all authors. Reviewed submitted version of manuscript: all authors. Approved the final version of the manuscript on behalf of all authors: Steven. Administrative/technical/material support: Steven. Study supervision: Steven. Creation of Fig. 2: Joswig.

\section{Correspondence}

David A. Steven, Department of Clinical Neurological Sciences, Division of Neurosurgery, London Health Sciences Centre, University Hospital, Rm. A10-323, 339 Windermere Rd., London, ON N6A5A5, Canada. email: david.steven@uwo.ca. 